\title{
Distal Fingertip Composite Graft in Amputation Salvaged through PGE-1 Assisted Treatment-A Case Report
}

\author{
Ho-Guen Chang ${ }^{1}$, Young-Jin Seo' ${ }^{2}$, and Jae-Hyuk Shin ${ }^{3 *}$ \\ ${ }^{1}$ Department of Orthopaedic Surgery, South Korea \\ ${ }^{2}$ Department of Orthopaedic Surgery, South Korea \\ ${ }^{3}$ Department of Orthopedic Surgery, South Korea
}

*Corresponding author: Jae-Hyuk Shin, Department of Orthopedic Surgery,

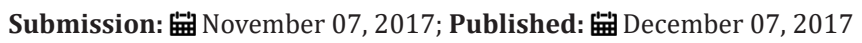

\begin{abstract}
51-Year-Old male patient injured distal phalanx amputation with distal phalanx fracture from being hit by a metal pipe. The fingertip amputation was oblique through the lunula of the nail. After clearing the wound margin, we directly reattached the composite graft to the proximal stump. PGE-1 was administered intravenously for one week which was switched to oral administration after that. The amputated fingertip began appearing mummified since postoperative one week. Regenerated vital tissue gradually substituted the mummified portion. After 12 months, regeneration was complete. With mild pulp hypotrophy and the finger regained working function in its original length. This case present distal fingertip composite graft is ultimately salvaged through mummification in combination treatment with PGE-1.
\end{abstract}

Keywords: Fingertip; Amputation; Composite graft; Salvage; PGE-1

\section{Introduction}

Treatment of the distal fingertip amputation remains in the debate due to inconsistent feasibility for vascular anastomosis. Besides direct microvascular anastomosis, alternative surgeries have been in-troduced such as Hirase's cooling principle [1], subcutaneous pocket graft [2], heteroditital transfer [3], and flap surgeries. These standard surgeries demand microsurgical infrastructure, as well as access to a medical institution within

emergent gold hours. Prostaglandin E1 (PGE-1) has been reported to contribute to dilating peripheral vessels and to increasing blood supply, and it has been widely used for the postoperative care of anastomosis and composite grafts [4]. However, once the graft is devitalized after a replantation surgery, most commonly adopted treatment solution is amputation. Here we present a salvage treatment technique for distal fingertip amputation in an adult, by composite graft assisted by PGE- 1 .

\section{Surgical technique presentation}
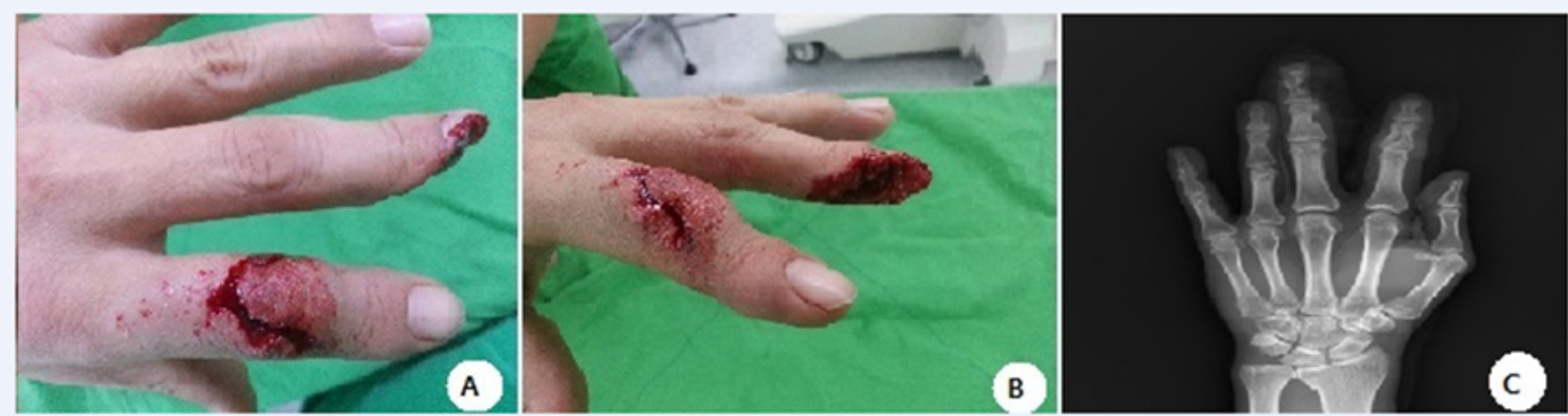

Figure 1: Initial amputation injury to the distal phalanx of the middle finger. The distal phalanx is obliquely amputated with a minimal portion of the nail remaining. The tendon attachments are preserved. (A and B) Preoperative clinical images, and (C) Radiograph. 
A 51 years old man injured distal phalanx amputation of the left middle finger, for being hit by a steel pipe. He had sustained diabetes mellitus (DM), and 30 pack-year of smoking history. The amputation was obliquely traversing in the distal phalanx through the lunula of the nail bed. Distal interphalangeal (DIP) joint capsule was torn. The $2^{\text {nd }}$ finger's flexor digitorum profundus and the extensor digitorum was intact (Figure1). The distal phalanx was fractured. The distal fingertip composite graft was reattached onto the stump. Distal phalanx fracture was internally fixated with a K-wire. Culture study revealed no active infection. Needle puncture to the fingertip showed negative capillary perfusion. PGE1 of Alprostadil was daily administered intravenously (Eglandin;

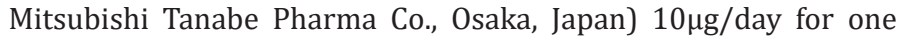
week followed by the oral tablet of Limaprost intake (Opalmon, $10 \mu \mathrm{g}$ tid). The tissue presented mummification appearance since postoperative one week (Figure 2).
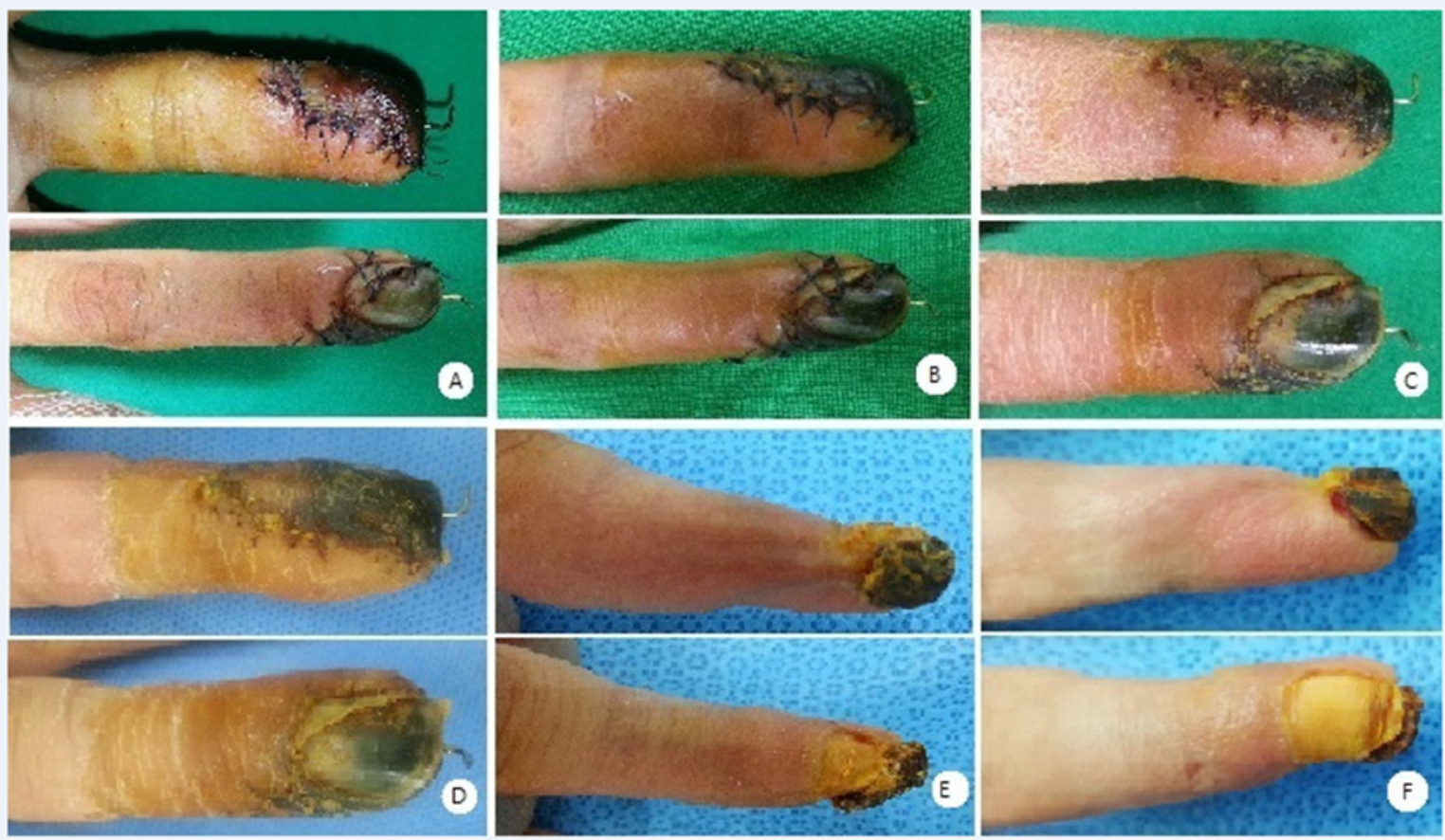

Figure 2: At one-week postoperatively, the middle finger began mummification appearance distal to the attachment. Prostaglandin-E1 (PGE-1) assisted conservative treatment has demonstrated wound healing, and the necrotic tissue was substituted by regenerated vital tissue. (A) Observation at one week, (B) three weeks, (C) five weeks, (D) eight weeks, (E) six months, and $(\mathrm{F})$ eleven months, postoperatively.

The mummified tissue was gradually substituted by degrees. The skin sensation was preserved, without paresthesia nor regeneration tissue from the vital border. After one year of conservative treatment, the tissue regeneration was complete (Figure 2). The active ROM of injured $3^{\text {rd }}$ finger DIP joint was 10-20

dysesthesia. The patient returned to active manual work utilizing the recovered finger (Figure 3).
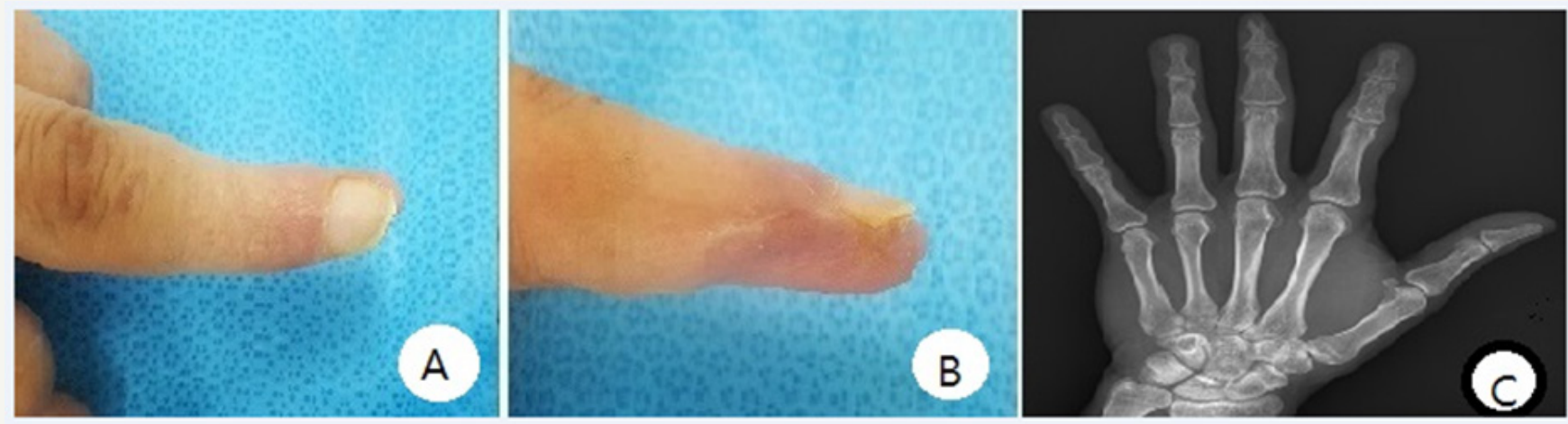

Figure 3: (A and B) At postoperative 12 months, the finger salvage was complete. Pulp hypotrophy is observed. Functional active range of movement is maintained to enable daily manual activities (see the main text). (C) Follow-up radiograph. 


\section{Discussion}

We observed delayed regeneration of amputated distal phalanx over early mummification following composite graft surgery, which was assisted by prostaglandin E1 administration. Composite graft alone has been reported to hardly survive in adult injuries, in contrast to young children. Survival of composite graft from amputation without microsurgical anastomosis has been reported. Cooling of the directly reattached amputated fingertip, without vascular anastomosis, enhances the graft survival. By lowering tissue metabolism in ice, the decay process would be minimized to sustain viability until vascular ingrowth forms an adequate channel [1-6]. Cooling principle enhances composite graft for the nose reconstruction [5].

Cap-technique in amputation around the lunula shortens soft tissue circumferentially on the proximal stump while preserving the germinal matrix $(>2 \mathrm{~mm})$ for growth-bud of the nail. The distal amputated finger is shortened of the bone, then repaired over proximal stump close to the vascular rich periosteal tissue. As the nail grows, the finger heals after normal appearance, but some shortening of the length is expected [7]. We directly reattached the bone and soft tissue with minimal wound margin preparation, thereby preserved the original length. Vascular smooth muscle regulates the microcirculation through excitatory and inhibitory substances [10].

PGE-1 antagonizes microvascular constrictive responsiveness by angiotensin, vasopressin, and constrictor catecholamines [10] PGE-1 shows diffuse dilating action upon all types of muscular micro-vessels, which decreases post arterial resistance to induce hypotensive effect [10]. PGE-1 application in hand angiography remarkably shows an increase of blood flow through the digital arteries via so dilatation leading to increasing of the volume of blood flow [13]. PGE-1 affects large vessels as well, as observed in the canine femoral artery [11]. Capillary permeability is increased by PGE-1 administration, synergizing vasodilatation in increasing oxygen supply [12]. Antithrombotic effect of PGE-1, supports microvascular replantation surgeries [4]. Ischemia-reperfusion injury of rat gastrocnemius muscle indicates enhanced blood flow with PGE-1 [14]. Microvascular anastomosis of ear amputation success-fully survives through PGE-1 administration [15].

If the digital arteries are damaged by distal phalanx amputation the blood supply mainly depends on collateral circulation. Without sufficient vasculature, the tissue became mummified. We administered PGE-1 intravenously for postoperative one week, then switched to oral regimen during following re-generation period. This case shows that even after early mummification following composite graft surgery on the distal phalangeal amputation, prudent, conservative treatment with patience, assisted by increased blood supply using PGE-1, can possibly lead to salvage regeneration of the composite graft. We speculate that while the mummified tissue function as the scaffold, the regeneration is encouraged by dilated peripheral vasculature and increased capillary permeability at the vital border.
This treatment harbors limitations as well. Tissue damage needs to be individually assessed for planning the treatment. The salvage regeneration requires rather prolonged treatment duration, demanding patience of the patient. Some pulp hypotrophy accompanies after healing through the mummification. Although this is a time requiring treatment, when microvascular surgery is not feasible either from damage status, or medical environmental cause, we suggest that this patient treatment may well be considered as a treatment option before deciding amputation.

\section{References}

1. Hirase Y (1997) Salvage of fingertip amputated at nail level: new surgical principles and treatments. Ann Plast Surg 38(2): 151-157.

2. Brent B (1979) Replantation of amputated distal phalangeal parts of fingers without vascular anastomoses, using subcutaneous pockets. Plast Reconstr Surg 63(1): 1-8.

3. Lee JY, Teoh LC, Seah VW (2006) Extending the reach of the heterodigital arterialized flap by cross-finger transfer. Plast Reconstr Surg 117(7): 2320-2328.

4. Rodriguez Vegas JM, Ruiz Alonso ME, Teran Saavedra PP (2007) PGE-1 in replantation and free tissue transfer: early preliminary experience. Microsurgery 27(5): 395-397.

5. Conley JJ, Vonfraenkel PH (1956) The principle of cooling as applied to the composite graft in the nose. Plastic and reconstructive surgery 17(6): 444-451.

6. Adani R, Marcoccio I, Tarallo L (2003) Treatment of fingertips amputation using the Hirase technique. Hand surgery : an international journal devoted to hand and upper limb surgery and related research. Journal of the Asia-Pacific Federation of Societies for Surgery of the Hand 8(2): 257-264.

7. Rose EH, Norris MS, Kowalski TA, Lucas A, Fleegler EJ (1989) The cap technique: non microsurgical reattachment of fingertip amputations. J Hand Surg Am 14(3): 513-518.

8. Bergstrom S, Eliasson R, von EU, Sjovall J (1959) Some biological effects of two crystalline prostaglandin factors. Acta physiologica Scandinavica 45(2-3): 133-144

9. Nakano J, McCurdy JR (1967) Cardiovascular effects of prostaglandin E1. The Journal of pharmacology and experimental therapeutics 156(3): 538-547.

10. Weiner R, Kaley G (1969) Influence of prostaglandin E1 on the terminal vascular bed. Am J Physiol 217(2): 563-566.

11. Campion T, Lynch TG, Kerr JC, Hobson RW (1986) Effects of prostacyclin injections and infusions on canine femoral hemodynamics. J Vasc Surg 3(3): 540-544.

12. Iversen VV, Reed RK (2002) PGE1 induced transcapillary transport of 51Cr-EDTA in rat skin measured by microdialysis. Acta physiologica Scandinavica 176(4): 269-274.

13. Levy JM, Joseph RB, Bodell LS, Nykamp PW, Hessel SJ (1983) Prostaglandin E1 in hand angiography. AJR Am J Roentgenol 141(5): 1043-1046

14. Rowlands TE, Gough MJ, Homer-Vanniasinkam S (1999) Do prostaglandins have a salutary role in skeletal muscle ischaemiareperfusion injury? European journal of vascular and endovascular surgery: the official journal of the European Society for Vascular Surgery 18(5): 439-444

15. Cavadas PC (2002) Supramicrosurgical ear replantation: case report. J Reconstr Microsurg 18(5): 393-395. 